\title{
Public Transport Passenger Attraction Using Policy Interventions for a Post-COVID Scenario
}

\author{
Sandra Kamar ${ }^{1} \cdot$ S. Shaheem ${ }^{2}$ B. Vinayaka ${ }^{1} \cdot$ Samson Mathew ${ }^{2}$
}

Received: 12 October 2021 / Accepted: 7 January 2022 / Published online: 1 March 2022

(c) The Author(s), under exclusive licence to Springer Nature Switzerland AG 2022

\begin{abstract}
Public transport (PT) ridership has seen a continual decline over the past years as more people are opting to use private vehicles, contributing to increased traffic congestions, road accidents, pollution, etc. The COVID-19 pandemic has exacerbated this problem, with official guidelines discouraging the use of PT systems to prevent contagion. Passenger attraction policies for a post-pandemic phase should be formulated by examining the changes in daily trips brought about by the COVIDinduced lockdowns. With data collected from employees working in Thiruvananthapuram city, the present study develops a post-lockdown mode-choice model using fuzzy logic programming to evaluate different policies to increase PT ridership. The policies such as introducing parking prohibition on major streets, reducing return-trip fares, improving PT coverage and supply, and early-bird pre-peak hour discounts were tested using sensitivity analysis and the choice model estimated a (private to PT) modal shift of 5.8\%, 6.9\%, 6.2\% and 6.2\%, respectively. It is concluded that passenger attraction policies should concentrate more on improving PT services than discouraging private modes to improve ridership.
\end{abstract}

Keywords COVID-19 $\cdot$ Fuzzy logic mode-choice model $\cdot$ Public transport policy

\section{Introduction}

In a globalised world where transportation of goods and people across countries and continents is vital, the international mass transit systems have had a huge role to play in turning COVID-19 into a world-wide pandemic. The official guidelines advice to maintain social distance and consequently discourages the use of PT as conditions in PT services promote contagion. In emerging economies like India, this has aggravated the already declining ridership rates in PT. The decline in PT patronage was hitherto attributed to factors

Sandra Kamar

sandra.kamaruddin@gmail.com; sandrakmr123@gmail.com

S. Shaheem

shaheems@yahoo.co.in

B. Vinayaka

vinayaknaikb@gmail.com

Samson Mathew

director.natpac@kerala.gov.in

1 School of Civil Engineering, REVA University, Bangalore, Karnataka, India

2 KSCSTE-National Transportation Planning and Research Centre, Thiruvananthapuram, Kerala, India such as the substandard services of the public transportation (PT) systems [4, 16], the psycho-socio-economic features of the commuters $[6,8,21,26]$, their travel characteristics, the appeal of private vehicle ownership and usage [20,22], and other miscellaneous factors. But with the COVID-19 pandemic, other factors like over-crowdedness [10, 24], fear of contagion and safety concerns come into play [3, 9, 11, 23].

This extensive modal shift from PT to private vehicles would result in more vehicles on the road, adversely affecting the highway system with increased traffic congestion, road accidents, etc. These issues further pave way for detrimental effects on the environment by means of air pollution through vehicular emissions, noise pollution, and other impacts. The menace of proliferating private vehicles extend to the economic sphere on account of the lives lost in road accidents, increased travel times, and other negative externalities. In order to resolve these issues, the PT sector must find ways to attract daily commuters who use private vehicles, persuading them to make a shift from private to public mode of transport. This brings up the question of passenger attraction policies for a post-pandemic world $[2,5,7,15,17$, 18]. The policies for passenger attraction towards PT services must be relevant and feasible and be tailored to suit the needs of its users. In this paper, a mode-choice model using 
fuzzy logic programming is developed using post-lockdown work-trip data collected from Thiruvananthapuram city, and several policies aimed at improving the ridership rates are tested using sensitivity analysis.

\section{Literature Review}

The COVID-19 induced lockdowns led to the suspension of operations of public transport buses, only to be resumed with restrictions after the lockdowns were lifted. Verma et al. [25] identified the impacts of COVID-19 on transportation infrastructure, while highlighting how the transportation system should be shaped to meet needs of the community post-COVID-19, and suggested a set of policy recommendations, strategies, and interventions to address the transportation issues in a post-COVID world. Implementation of staggered work hours, staggered business schedules along with prioritizing the movement of unskilled and low-skilled workgroup were emphasised and infrastructural modifications including temporary Bus-Priority Lanes, temporary bus stations, markings for passengers to maintain a safe distance and implementation of smart-cards or QR-based ticketing, etc. were suggested [25]. Jung [12] explored how epidemics impact ridership on public transportation in the background of the outbreak of the Middle East respiratory syndrome coronavirus (MERS) in South Korea that occurred from May 2015 to July 2016. The results revealed that middle-income class increased their gasoline expenditures and lowered spending on public transportation, while high income class, already had the largest/smallest expenditures on gasoline/public transportation, whereas the low-income class, being captive riders, failed to reduce public transit ridership during the outbreak and maintained the strongest commitment to public transportation [12].

People's intentions to use public transport was explored by Abdullah et al. [1] using three different models (during the pandemic, while following the necessary precautions and while experiencing COVID-19 symptoms). The results suggested that latent attributes like "attitudes", "responsibility" and "difficulty" had an inverse relationship whereas, "awareness" had a direct relationship with the intentions to use public transport during the pandemic, and from these results a policy interventions framework was proposed to promote the safe use of various public transport modes, particularly in developing regions [1].

Studies on improving the prediction accuracy of the mode-choice models has led to employing soft techniques like fuzzy logic programming to predict mode choice of travellers. Kumar et al. [14] applied the fuzzy logic technique in which the mode-choice model provides the estimated modal split at the specified work centres in Delhi and concluded that the fuzzy logic-based mode-choice model offers significant flexibility in evaluating any kind of sensitive policy for public transport system. Kedia et al. [13] attempted to analyse the transit choice behaviour of urbanites, with the help of fuzzy rule-based mode-choice model, considering Surat, an Indian metropolitan city in the state of Gujarat as the study area, and concluded that fuzzy based models overcome the shortcomings of Logit models as travellers' perceptions of accessibility attributes can be better expressed in linguistic form. Pulugurta et al. [19] applied the concept of fuzzy logic for modelling mode choice in Port Blair city, India, and compared the results with traditional MNL model, concluding that the fuzzy logic model gave better prediction accuracy in comparison to the traditional MNL model as it was better able to capture and incorporate the human knowledge and reasoning into mode-choice behaviour, and further, selected transport policies were evaluated using the model.

The literature review suggests that no studies have been made on how COVID-19 has affected the public transportation system in medium-sized cities. And while fuzzy logicbased mode-choice models have been found to be suitable to analyse policies, there is no existing research that makes use of the fuzzy logic-based mode-choice model for COVIDrelated research. With insights gained from the review, the study objectives and methodology were formulated.

\section{Methodology}

The objective of this study is to identify the policies that attract potential passengers while retaining the existing users and improving the service quality of PT for a post-COVID scenario. This section explains the methodology followed to achieve this objective. The literature review helped in formulation of objectives and selection of the study area, and for data collection a survey questionnaire that included all the necessary information to carry out this study was designed. The data collected from the survey was cleaned to prepare it for further analysis. Descriptive statistical analysis and cross-tabulation analysis was carried out on the cleaned data as a precursor to further analysis and to get an overall idea of the nature of the data.

The post-lockdown travel parameters were used to develop a post-lockdown mode-choice model using fuzzy logic programming. To check the application of the model, several policies were formulated, and an expert opinion survey was carried out to select policies of high relevance and feasibility and the effects of the selected policies were tested using sensitivity analysis. Further, a comparison between the estimated modal split and the modal split induced by the selected policies were made to evaluate the effectiveness of these policies in increasing PT ridership. 


\section{Study Area}

The present study focuses on work trips in emerging economies. A medium-sized city with high working population was set as the criteria for the selection of study area. Since most cities in India are medium-sized, the results can be applied elsewhere in the country. Consequently, Thiruvananthapuram City in the state of Kerala, India was selected. Thiruvananthapuram, with a population of 957,730 , is the most populous city in Kerala as per the 2011 census, in addition to being the state capital. The work participation rate of Thiruvananthapuram district, as reported in the 2011 census, is $37 \%$. The transportation infrastructure is well developed, and the city can be traversed through roadway and railway. The state-run Kerala State Road Transport Corporation (KSRTC) dominates the public bus services in the city, while private bus services are also available. With insights from the relevant literature, the questionnaire was designed to collect information regarding pre-COVID and post-lockdown trip characteristics, socio-economic characteristics, data related to the pandemic situation and Likert scale ratings on the sustainable transport preferences of the respondents.

\section{Data Collection}

For the current study, data were collected from employees at their respective workplaces in different parts of Thiruvananthapuram city. The main data collection points were Pattom, Palayam, Keshavadasapuram, Statue, Ulloor, and Nedumangad. After data entry and subsequent data cleaning, a total of 469 samples were taken for analysis. Descriptive statistical analysis on the data revealed the demographic features of the respondents, with $45.42 \%$ male respondents and $54.58 \%$ female respondents. $32.84 \%$ belonged to the $31-40$ age group, followed by $30.49 \%$ respondents in the 41-50 age group, and the 21-30 group were found to be $23.24 \%$, among others. Taking marital status, $83.58 \%$ were married, $15.57 \%$ unmarried and $0.85 \%$ responded to others. The data shows a majority response of $45.42 \%$ for the household size of four individuals, followed by $30.06 \%$ for more than four members, and $17.48 \%$ with three members, among others. The data shows that $49.68 \%$ has an undergraduate degree and $18.55 \%$ respondents held a post-graduate degree. The monthly personal income of $30.06 \%$ respondents was between 20,000 and 30,000 rupees per month, followed by $23.45 \%$ earning 10,000 to 20,000 , while $22.81 \%$ had between 30,000 to 40,000 per month. The percentage of working members to total members in the household was $25-50 \%$ for $45.20 \%$ of the respondents. $88.91 \%$ were found to own at least one private vehicle. The post-lockdown work/business trip frequencies were analysed and $95.94 \%$ was found to be daily trips and $2.137 \%$ alternate-day trips.
The post-lockdown mode of travel for these work-trips were $54.06 \%$ two-wheelers, $19.01 \%$ PT buses, $15.60 \%$ private cars and the rest by walk, cycle, passenger auto, taxi, train, and institution vehicle.

\section{Fuzzy Logic}

The term "fuzzy logic" was first coined by Lotfi Zadeh with the 1965 'fuzzy set theory' proposal. As the term 'fuzzy' suggests, this technique deals with the concept of partial truth, where the truth value may range anywhere between completely true and completely false. Fuzzy logic makes it possible to take in an open, imprecise spectrum of data, and give out accurate results. It is a form of artificial intelligence whose method of reasoning resembles human reasoning, and therefore is ideal in developing a mode-choice model. The mode-choice model is developed in the MATLAB software, which provides a Graphical User Interface to carry out the modelling with minimal coding.

\section{Membership Functions}

The input used in conventional mode-choice models are defined by crisp sets. For example, $12 \mathrm{~km}$ distance would come under medium, but $13 \mathrm{~km}$, which is only one kilometre longer would be excluded from the medium set. Whereas, in fuzzy logic, the input data, which usually are crisp values, are fuzzified using membership functions (MF), which assigns a value ranging between 0 and 1 , called 'degree of membership' or 'membership value' for each input within a certain range. Therefore, 13 will be assigned a certain degree of membership under the medium set, as illustrated in Fig. 1.

The MFs are of different types such as triangular, trapezoidal, Gaussian, etc. and they can be set to overlap as shown in Fig. 2.

A fuzzy set $\tilde{A}$ in the universe $U$ can be defined as a set of ordered pairs and it can be represented mathematically as:

$\tilde{A}=\left\{\left(y, \mu_{\tilde{A}}(y)\right) \mid y \in \mu\right\}$,

where, $\mu_{\tilde{A}}(y)=$ membership of $y$ in $\tilde{A}, y=$ element in $\tilde{A}$, $\mu_{\tilde{A}}(y) \in[0,1]$.

The fuzzified inputs are fed into a fuzzy inference engine containing "if-then" rules in which product-sum gravity technique is used to generate output in the linguistic form. Since these outputs cannot be used directly, they are de-fuzzified to give a crisp output. In summary, the system takes crisp values as inputs, which are processed for fuzzification as per MFs, and then analysed based on fuzzy if-then rules in the fuzzy inference engine to generate output in the linguistic form, and then de-fuzzified to give crisp output (refer Fig. 3). 
Fig. 1 Difference between crisp set and fuzzy set
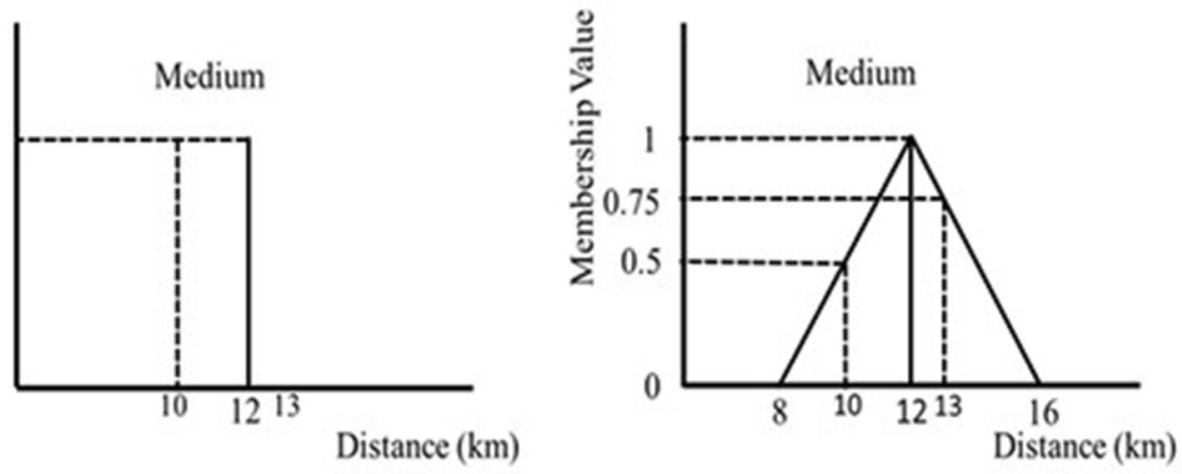

Fig. 2 Membership function plots

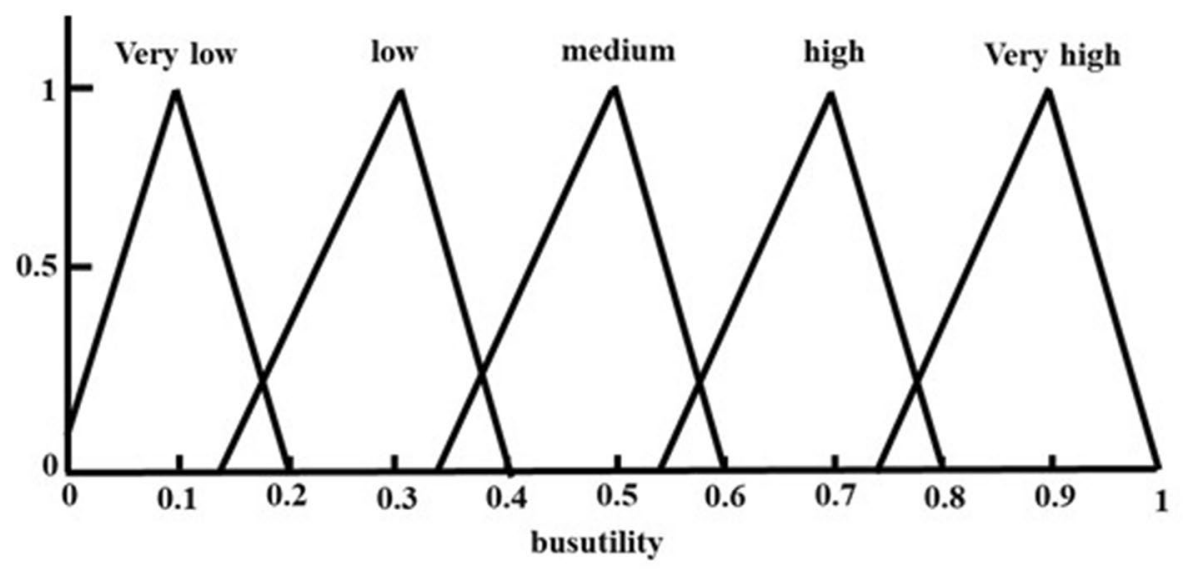

Fig. 3 Fuzzy logic system structure

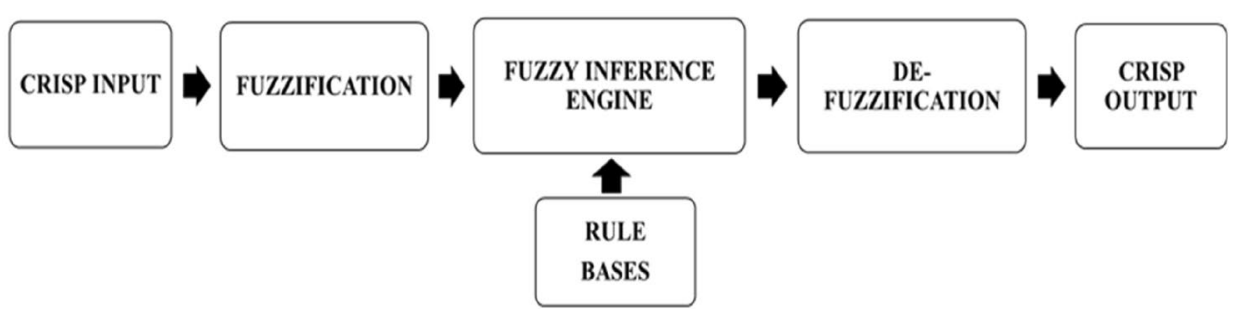

\section{Rule Bases}

Rule bases are one of the most important parts of the fuzzy logic system structure as the output given by the model depends on them. The if-then rule bases specify the relationship between the input parameters and output parameters. The rule bases in fuzzy logic system are in the following format:

IF (input A) is (Level of MF) and/or (input B) is (Level of MF), THEN (output X) is (Level of MF) and (output Y) is (Level of MF).

There can be any number of inputs, any number of outputs, and any number of MF levels. The accuracy of fuzzy logic systems depends heavily on the if-then rules that are fed into the fuzzy inference engine. A good practice to set the fuzzy rule bases is to study the results of descriptive statistical analysis of the data carefully and adapt the findings into the rules, and this method has been adopted while setting the rules for this study. To increase the accuracy of the model, modification of either the rule bases or the MFs on a trial-and-error basis is found to be effective.

\section{Fuzzy Logic Mode Choice Model}

For the present study, a fuzzy logic mode-choice model was developed using six input parameters: time, travel cost, income, gender, percentage of working to total members in the household, and age. The shape of all the membership functions was set as triangular, except for the gender variable which was set as trapezoidal (gender does not require fuzzification). The utility for five modes including Car, TW, Bus, 
Walk, and Auto were considered. The membership levels set for the input and output variables are as shown in Table 1.

The input variables were taken in a combination of two and 300 rules were formulated for each mode. A total of 4800 rule bases were set to make the post-lockdown fuzzy logic-based mode-choice model.

The prediction accuracy of the calibrated model with $70 \%$ data for the post-lockdown fuzzy logic mode-choice model is illustrated in Table 2. The calibrated post-lockdown fuzzy logic model predicted modes like walk, two-wheeler, auto, car, and bus with a prediction accuracy of about $80 \%$.

The calibrated model was further validated to check for prediction accuracy as presented in Table 3 . The validated post-lockdown fuzzy logic model had a prediction accuracy of about $57 \%$. But as given in the tables above, the model fails to predict WALK and AUTO modes. This is because out of the 469 respondents, only 21 persons (4.4\%) used the mode WALK, and only 13 persons (2.7\%) used AUTO. The sample size constraints coupled with a prediction accuracy of about $80 \%$ explains why these modes were not predicted.

\section{Sensitivity Analysis}

Transportation policies are part of an overall process of intervention aimed at making effective decisions concerning the allocation of transport resources for improvement and maintenance of transportation services. The policies for passenger attraction towards PT services must be formulated to mould the system into an effective and efficient network, catering to suit the needs of its users.

A total of 25 policies were formulated with regards to improving the PT services, depressing the ownership, and discouraging the usage of private vehicles. An expert
Table 1 Membership-level ranges

\begin{tabular}{|c|c|c|}
\hline Parameter & Level of MF & Range \\
\hline \multirow[t]{5}{*}{ Time (in min) } & Very low & $\left(\begin{array}{lll}0 & 15 & 30\end{array}\right)$ \\
\hline & Low & $\left(\begin{array}{lll}10 & 30 & 50\end{array}\right)$ \\
\hline & Medium & (30 60 90) \\
\hline & High & $\left(\begin{array}{llll}60 & 80 & 100\end{array}\right)$ \\
\hline & Very high & $\left(\begin{array}{llll}80 & 110 & 120\end{array}\right)$ \\
\hline \multirow[t]{5}{*}{ Income group ${ }^{\mathrm{a}}$} & Very low & $\left(\begin{array}{lll}0 & 0.8 & 1.5\end{array}\right)$ \\
\hline & Low & $\left(\begin{array}{lll}0.5 & 1.5 & 2.5\end{array}\right)$ \\
\hline & Medium & $(1.534 .5)$ \\
\hline & High & $(3.54 .55 .5)$ \\
\hline & Very high & $(4.55 .56 .5)$ \\
\hline \multirow[t]{5}{*}{ Age } & Very young & $\left(\begin{array}{lll}15 & 20 & 25\end{array}\right)$ \\
\hline & Young & $(203035)$ \\
\hline & Middle age & $(304050)$ \\
\hline & Senior age & $(455560)$ \\
\hline & Elder & $\left(\begin{array}{l}55 \\
62\end{array} 69\right)$ \\
\hline \multirow[t]{5}{*}{ Percentage of working members to total members in the household } & Very low & $\left(\begin{array}{lll}0 & 10 & 25\end{array}\right)$ \\
\hline & Low & $(52545)$ \\
\hline & Medium & $(255075)$ \\
\hline & High & $(507595)$ \\
\hline & Very high & $\left(\begin{array}{llllll}80 & 100 & 100\end{array}\right)$ \\
\hline \multirow[t]{5}{*}{ Travel cost } & Very low & $\left(\begin{array}{lll}0 & 0 & 20\end{array}\right)$ \\
\hline & Low & $(53060)$ \\
\hline & Medium & (35 65 100) \\
\hline & High & $\left(\begin{array}{lllllll}75 & 100 & 130\end{array}\right)$ \\
\hline & Very high & $\left(\begin{array}{llll}100 & 150 & 200\end{array}\right)$ \\
\hline \multicolumn{3}{|c|}{$\begin{array}{l}\text { aa Grouping of income: } 1-\leq 10,000,2-10,000-20,000,3-20,000-30,000,4-30,000-40,000,5-50,000- \\
100,000,5-\geq 100,000\end{array}$} \\
\hline \multirow[t]{5}{*}{ Bus utility/car utility/walk utility/TW utility/auto utility } & Very low & $\left(\begin{array}{llll}0 & 0.1 & 0.2\end{array}\right)$ \\
\hline & Low & $\left(\begin{array}{lll}0.15 & 0.3 & 0.4\end{array}\right)$ \\
\hline & Medium & $\left(\begin{array}{llll}0.35 & 0.5 & 0.6\end{array}\right)$ \\
\hline & High & $\left(\begin{array}{llll}0.55 & 0.7 & 0.8\end{array}\right)$ \\
\hline & Very high & (0.75 0.9 1) \\
\hline
\end{tabular}


Table 2 Calibrated prediction table for post-lockdown fuzzy logic model

\begin{tabular}{lllllll}
\hline Post-lockdown calibrated model & & & & \\
\hline & \multicolumn{2}{l}{ Observed } & & & Total Estimated \\
\cline { 2 - 6 } & WALK & TW & AUTO & CAR & BUS & \\
\hline Estimated & & & & & & \\
WALK & $\mathbf{0}$ & 0 & 1 & 0 & 0 & 1 \\
TW & 1 & $\mathbf{1 8 9}$ & 4 & 17 & 16 & 227 \\
AUTO & 1 & 0 & $\mathbf{0}$ & 0 & 0 & 1 \\
CAR & 2 & 8 & 1 & $\mathbf{3 6}$ & 7 & 54 \\
BUS & 5 & 1 & 2 & 0 & $\mathbf{3 5}$ & 43 \\
Total observed & 9 & 198 & 8 & 53 & 58 & $\mathbf{3 2 6}$ \\
Prediction accuracy & $((\mathbf{0}+\mathbf{1 8 9}+\mathbf{0}+\mathbf{3 6}+\mathbf{3 5}) / \mathbf{3 2 6}) \times \mathbf{1 0 0}=\mathbf{7 9 . 7 5 \%}$ & & \\
\hline
\end{tabular}

The numbers in bold inside the matrix are the correct predictions, the calculation at the end shows the prediction accuracy which is the main result derived out of the table

\begin{tabular}{|c|c|c|c|c|c|c|}
\hline \multicolumn{7}{|c|}{ Post-lockdown validated model } \\
\hline & \multicolumn{5}{|c|}{ Observed } & \multirow[t]{2}{*}{ Total estimated } \\
\hline & WALK & TW & AUTO & CAR & BUS & \\
\hline \multicolumn{7}{|l|}{ Estimated } \\
\hline WALK & $\mathbf{0}$ & 0 & 1 & 0 & 0 & 1 \\
\hline TW & 5 & 58 & 3 & 5 & 26 & 97 \\
\hline AUTO & 0 & 0 & $\mathbf{0}$ & 0 & 1 & 1 \\
\hline CAR & 2 & 5 & 1 & 11 & 3 & 22 \\
\hline BUS & 5 & 3 & 0 & 1 & 11 & 20 \\
\hline Total observed & 12 & 66 & 5 & 17 & 41 & 141 \\
\hline Prediction accuracy & \multicolumn{6}{|c|}{$((0+58+0+11+11) / 141) \times 100=56.73 \%$} \\
\hline
\end{tabular}

The numbers in bold inside the matrix are the correct predictions, the calculation at the end shows the prediction accuracy which is the main result derived out of the table
Table 3 Validated prediction table for post-lockdown fuzzy logic model opinion survey was carried out, asking the experts in the industry to rate the relevance and feasibility of these policies on a scale of 1-5 (1-not relevant/feasible, 2-less relevant/ feasible, 3-neutral, 4-relevant/feasible, 5-very relevant/ feasible). The policies having the highest rating for both relevance and feasibility were selected from a total of 52 responses received. The selected policies and their ratings of relevance and feasibility are shown in Fig. 4. The following policies were chosen for sensitivity analysis:

- Policy 1: Improving PT coverage and supply, reducing travel time by $5 \%$.

- Policy 2: Parking prohibition on major streets, reducing congestion and PT travel time by $10 \%$.

- Policy 3: Early bird pre-peak hour discount of 5\%.

- Policy 4: Reducing fare by $10 \%$ for return journey for passengers purchasing two-way tickets.
- Policy 5: Combination of Policy 2 and Policy 4.

- Policy 6: Combination of Policy 1 and Policy 3.

Other relevant policies like single ticketing system and operating limited stop bus services, having equally high relevance and feasibility rating, can be considered to have the same effect on travel cost and travel time as Policy 4 and Policy 2, respectively. Policy 3 is especially relevant to this study considering its potential to reduce overcrowding during peak hours.

The five policies were applied to the post-lockdown fuzzy logic mode-choice model. Table 4 contains the results of the policy sensitivity analysis applied on post-lockdown fuzzy logic mode-choice model. The results suggest that Policies 4 and 5 will increase PT bus ridership by $6.9 \%$, while Policy 2 induces the lowest shift of $5.8 \%$. 


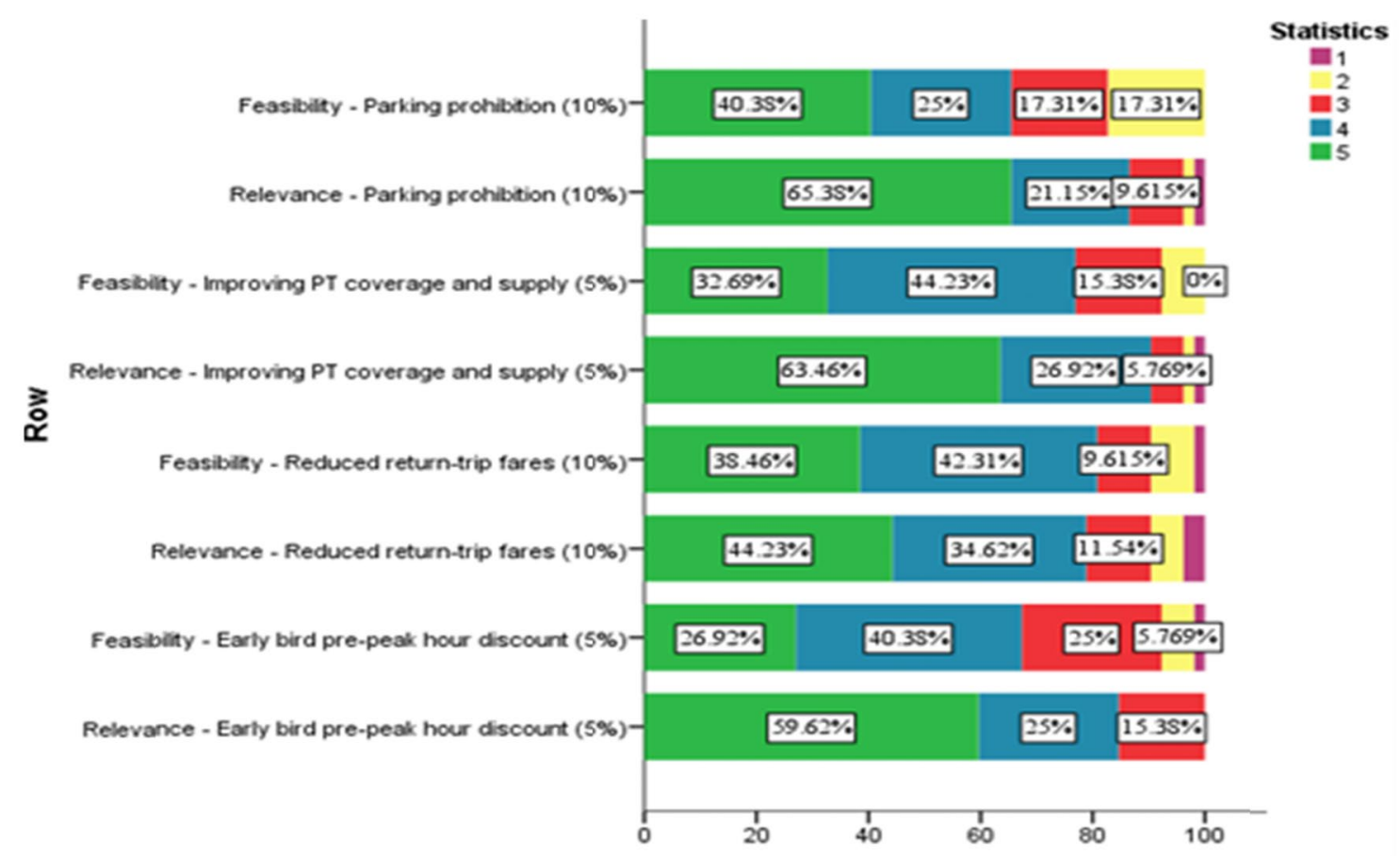

Fig. 4 Policy ratings

Table 4 Post-lockdown modal split after policy sensitivity analysis

\begin{tabular}{llllllll}
\hline MODE & Estimated & Policy 1 & Policy 2 & Policy 3 & Policy 4 & Policy 5 & Policy 6 \\
\hline Private vehicles & 85.9 & 79.7 & 80.1 & 79.7 & 79.0 & 79.0 & 79.7 \\
Public transport & 14.1 & 20.3 & 19.9 & 20.3 & 21.0 & 21.0 & 20.3 \\
Shift to PT & & $\mathbf{6 . 2 \%}$ & $\mathbf{5 . 8 \%}$ & $\mathbf{6 . 2 \%}$ & $\mathbf{6 . 9 \%}$ & $\mathbf{6 . 9 \%}$ & $\mathbf{6 . 2 \%}$ \\
\hline
\end{tabular}

The shift percentage from private to public transport is the main takeaway (in bold)

\section{Conclusion}

The present study attempts to understand the relevant policies that may induce a modal shift from private mode of transport to public mode of transport for a post-COVID scenario. The fuzzy logic model developed for this study has a prediction accuracy of about $80 \%$ and validation accuracy of $57 \%$.

The results of the policy sensitivity analysis of the postlockdown fuzzy logic mode-choice models suggests that all the policies considered may increase the PT ridership and reduce private vehicle usage. Policy 1 , which aims to improve PT coverage and supply, thereby reducing travel time by $5 \%$ is found to reduce private vehicle usage by $6.2 \%$. A reduction of $5.8 \%$ is observed when Policy 2 is introduced, where parking prohibition on major streets aims to reduce congestion and PT travel time by $10 \%$. It is also observed that Policy 3, which implements early-bird pre-peak hour discount of $5 \%$ impact the reduction of private vehicles by
$6.2 \%$. Policy 4 , aimed at reducing travel cost by $10 \%$ for the return journey of passengers purchasing two-way tickets is found to reduce private vehicle usage by $6.9 \%$. Policy 5 , which is a combination of the policies 2 and 4 reduces both travel time and travel cost by $10 \%$ and gives similar results as Policy 4. A shift of 6.2\% is observed for Policy 6, which is a combination of Policies 1 and 3 .

From the results obtained, it can be seen that a reduction in travel cost induces a higher modal shift (Policy 4) from private to public mode of transport. This could be attributed to the purpose of the trip selected for this study, i.e., work trips. It can be inferred that the commuters give more importance to travel cost when it comes to work trips, especially taking into consideration the financial crisis caused by COVID-19.

Since this study is limited to work-trips in a mediumsized city, future research can investigate different trip purposes. Another limitation is that the fuzzy logic modechoice model developed in this study does not consider 
the latent attributes that affect the PT ridership and consequently, does not test the effect of policies in improving PT ridership as a consequence of enhancing them. Studies in the future can address these limitations by including latent attributes in a fuzzy logic-based mode-choice model for a post-pandemic scenario and by comparing the findings with pre-COVID results.

Acknowledgements The authors are grateful to KSCSTE-NATPAC for all the support provided throughout the project, amidst the COVID19 pandemic situation. The authors acknowledge the opportunity provided by the 6th Conference of the Transportation Research Group of India (CTRG-2021) to present the work that formed the basis of this manuscript.

\section{Declarations}

Conflict of interest The authors declare that they have no conflict of interest.

\section{References}

1. Abdullah M et al (2021) Exploring the traveler's intentions to use public transport during the COVID-19 pandemic while complying with precautionary measures. Appl Sci 11:3630

2. Adnan et al (2020) Examining impacts of time-based pricing strategies in public transportation: a study of Singapore. Transp Res Part A 140:127-141

3. Arellana $\mathbf{J}$ et al (2020) COVID-19 outbreak in Colombia: an analysis of its impacts on transport systems. J Adv Transp 2020:8867316. https://doi.org/10.1155/2020/8867316

4. Chiou et al (2015) Factors affecting public transportation usage rate: geographically weighted regression. Transp Res Part A 78:161-177

5. Danesh A, Ma W, Wang L (2019) Policy solutions for transportation-related problems in Kabul: lessons from major cities of China and India. In: 19th COTA international conference of transportation $\odot$ ASCE

6. Efthymiou D et al (2018) Factors affecting bus users' satisfaction in times of economic crisis. Transp Res Part A Policy Pract 114:3-12

7. Evangelinos (2018) Pricing workplace parking via cash-out: effects on modal choice and implications for transport policy. Transp Res Part A 113:369-380

8. Fu, Juan (2017) Exploring the psychosocial factors associated with public transportation usage and examining the "gendered" difference. Transp Res Part A 103:70-82

9. Gutiérrez A, Miravet D, Domènech A (2020) COVID-19 and urban public transport services: emerging challenges and research agenda. Cities Health. https://doi.org/10.1080/23748834.2020. 1804291
10. Haywood et al (2017) Crowding in public transport: who cares and why? Transp Res Part A 100:215-227

11. Hendrickson C, Rilett LR (2020) The COVID-19 pandemic and transportation engineering. Ed J Transp Eng Part A Syst 146(7):01820001

12. Jung $H$ (2020) The impact of an epidemic on transit ridership. $\mathbf{J}$ Public Transp 22(2):1-15

13. Kedia AS, Sowjanya D, Salini PS, Jabeena M, Katti BK (2017) Transit shift response analysis through fuzzy rule based-choice model: a case study of Indian metropolitan city. Transp Dev Econ 3:8. https://doi.org/10.1007/s40890-017-0038-9

14. Kumar M, Sarkar P, Madhu E (2013) Development of fuzzy logic based mode choice model considering various public transport policy options. Int J Traffic Transp Eng 3(4):408-425

15. Lee, Yeh (2019) Causal effects between bus revenue vehiclekilometres and bus ridership. Transp Res Part A 130:54-64

16. Li B, Cao JX (2018) Analysis of influencing factors on satisfaction degree for urban public transit service based on structural equation model. In: 18th COTA international conference of transportation professionals $($ ) ASCE

17. Liu et al (2018) Effects of vehicle restriction policies: analysis using license plate recognition data in Langfang, China. Transp Res Part A 118:89-103

18. Liu et al (2020) Noncompliance behaviour against vehicle restriction policy: a case study of Langfang, China. Transp Res Part A 132:1020-1033

19. Pulugurta S, Arun A, Errampalli M (2013) Use of artificial intelligence for mode choice analysis and comparison with traditional multinomial logit model. In: 2nd Conference of transportation research group of India

20. Shi J, Jian MY (2015) Analysis of car ownership growth trend in China under the purchasing restriction policy. In: 15th COTA international conference of transportation professionals (C) ASCE

21. Sun, Duan (2019) Modelling passengers' loyalty to public transit in a two-dimensional framework: a case study in Xiamen, China. Transp Res Part A 124:295-309

22. Tao et al (2019) The role of car ownership in attitudes towards public transport: a comparative study of Guangzhou and Brisbane. Transp Res Part F 60:685-699

23. Tirachini, Cats (2020) COVID-19 and public transportation: current assessment, prospects, and research needs. J Public Transp 22(1): $1-21$

24. Tirachini et al (2017) Estimation of crowding discomfort in public transport: results from Santiago de Chile. Transp Res Part A 103:311-326

25. Verma et al (2020) Towards a sustainable transportation system for the COVID 19 recovery period. Un-published White Paper, Transportation Engineering Laboratory, Department of Civil Engineering, IISc 26 Bangalore, India

26. Vicente, Reis (2018) Ex-regular users of public transport: their reasons for leaving and returning. J Public Transp 21(2):101-116

Publisher's Note Springer Nature remains neutral with regard to jurisdictional claims in published maps and institutional affiliations. 